\title{
Carrots and Sticks: Prizes and Punishments in Contests
}

\author{
Benny Moldovanu, Aner Sela, Xianwen Shi*
}

March 23, 2010

\begin{abstract}
We study optimal contest design in situations where the designer can reward high performance agents with positive prizes and punish low performance agents with negative prizes. We link the optimal prize structure to the curvature of distribution of abilities in the population. In particular, we identify conditions under which, even if punishment is costly, punishing the bottom is more effective than rewarding the top in eliciting effort input. If punishment is costless, we study the optimal number of punishments in the contest.
\end{abstract}

Jel Classification: D44, D82, J31, J41.

Keywords: Contests, All-pay auctions, Punishments, Order Statistics.

\footnotetext{
*We would like to thank two anonymous referees for helpful comments. Shi thanks a research grant from SSHRC for financial support. Moldovanu: Department of Economics, University of Bonn, Lennestr. 37, 53113 Bonn, Germany; e-mail: mold@uni-bonn.de. Sela: Department of Economics, Ben Gurion University, Beer Sheva 84105, Israel; e-mail: anersela@bgu.ac.il. Shi: Department of Economics, University of Toronto, 150 St. George Street, Toronto, ON M5S 3G7, Canada; e-mail: xianwen.shi@utoronto.ca.
} 


\section{Introduction}

Contests are widely used to model political lobbying, sports competitions, job promotions, and $R \& D$ races. In most cases, the contest's organizer has some control over the structure of rewards and punishments: teachers determine who passes and who fails the exam, a company sets rules about promotions and layoffs, and sport governing bodies choose the number of teams advancing to a higher league and the number of those relegated to a lower one.

In this paper we characterize the optimal prize structure in a contest where the designer can use both rewards (carrots) and punishments (sticks) in order to motivate participants to exert effort 11 Although both rewards and punishments are part of many existing incentive contracts, the literature on contests focused on the incentive role of rewards because a contest that uses some punishments can be replicated by one that uses only rewards and yields the same incentives. But, this equivalence breaks down if the contest designer faces a budget constraint and if both rewards and punishments are costly. The main focus of the present paper is to study contest design when the designer is budget constrained and it is costly to punish or reward players.

Costly punishments appear in many situations. For example, in labor tournaments a firm usually incurs costs when firing workers who perform poorly (e.g., worker compensation, production disruption, loss of clients), and usually face search and replacement costs (e.g., think about a faculty denying tenure to some candidates). In other instances, punishments are less costly to implement (e.g., relegation in sport tournaments), and we also briefly deal with this set-up.

We assume here that the designer has a limited budget, which means that costly punishments cannot be arbitrarily high. Subject to her budget constraint, the designer determines the number and size of prizes/punishments in order to maximize the participants' expected total effort. In particular, some prizes can be positive (rewards) or negative (punishments). Given the prize structure, players choose their effort levels in order to maximize their expected payoff.

It is important to note that in a contest with $n$ participants the marginal effect of each of $n$ prizes on the participants' total effort is different, and can be either positive or negative. If the marginal effect of the $k$-th prize is positive, its value given a budget constraint should be positive (reward) or zero, and if the marginal effect is negative, its value should be negative (punishment) or zero. Thus, given that the contest designer has a limited budget, he will prefer to distribute the entire prize sum to the prize with the highest marginal effect on the participants' total effort

\footnotetext{
${ }^{1}$ See Arvey and Ivancevich (1980) for a comprehensive discussion about the use of punishments in organizations.
} 
relative to its cost.

In this paper we show that the relative effectiveness of punishments compared to rewards crucially depends on the curvature of the distribution of player abilities. Exploiting results about normalized spacings among order statistics developed by Barlow and Prochan (1966), we first revisit one of the main results in Moldovanu and Sela (2001): if punishment is not feasible, the optimal prize structure offers a single reward to the top performer. If rewards are not feasible, we then show that the designer should only punish the player with the lowest performance if the distribution of abilities has an increasing hazard (or failure) rate. If this last condition is not satisfied, more punishments may be optimal.

If the designer can punish as well as reward, the optimal prize structure will depend on the relative marginal cost of punishments, and on the distribution of the players' abilities. For example, if the marginal cost of punishments is the same as the marginal cost of rewards, it is optimal to punish the worst performing player if the distribution of abilities is convex. If that player is punished, even high types will work hard to avoid performing worst because they know that there is a relatively high probability that competitors also have high types. With a constant marginal cost of punishment, rewards and punishments cannot co-exist in the optimal prize structure because the only thing that matters is the comparison of the marginal benefit of top reward with the marginal benefit of punishments. Punishment and rewards may co-exist in our setting if punishing the bottom player is more cost effective than rewarding the top player and punishments have a fixed value which cannot be optimized upon ${ }^{2}$.

Costless punishments are, of course, more attractive to the designer. We first look at the case where players have no exit option, and we show that the optimal number of punishments with a fixed value is decreasing in the convexity of the ability distribution. Intuitively, when the distribution is more convex, there are relatively more high ability players. In order to motivate participants to work hard, the designer should differentiate between players with moderate performances and low performances, rather than punishing all of them.

In many situations players have outside options, and thus they may choose not to participate in the contest. Thus, the designer needs to satisfy an individual rationality constraint (chosen here to be interim: the expected payoff of a player, conditional on his ability, must be nonnegative). We show that punishments continue to be effective even in this case since they allow the designer to

\footnotetext{
${ }^{2}$ Alternatively, if punishment costs are convex in the punishment size, then co-existence is possible even without this last restriction.
} 
exclude low ability participants, akin to entry fees or reserve prices.

In this paper, we restrict our attention to the case where the contest designer aims to maximize the expected total effort exerted by all contestants. This seems consistent with various contests in labor markets. In other applications, the designer may have different objectives. For example, in sporting competitions the designer may be interested only in the performance of the top player, or in having a close competition (see an analysis of 'suspense' in Chan, Courty and Li, forthcoming). In principle, the present framework can be used to analyze contest design problems with other goals, but the results will obviously depend on the particular setting and goal.

The two papers most closely related to the present research are Moldovanu and Sela (2001) and Moldovanu, Sela and Shi (2007). The first develops an all-pay auction framework with incomplete information in order to study the optimal allocation of several non-negative prizes (rewards) in contests where the designer has a fixed budget 3 . Moldovanu, Sela and Shi (2007) apply the all-pay auction framework to the analysis of the incentive effects of social status in organization design 4 . In their model, contestants who perform poorly have a low social status, and it is shown that the designer can take advantage of the agents' status concern in order to induce high effort. That paper did not study, however, the contest design problem with a general structure of rewards and punishments.

Our paper is also related to the large literature on tournaments, initiated by Lazear and Rosen (1981). That literature has shown how prizes based on rank-orders of performance can be effectively used to provide incentives in labor tournaments (see also Green and Stokey (1983), and Nalebuff and Stiglitz (1983)). The labor tournament model has been extended to analyze political lobbying and research contests. For example, Che and Gale (1998) use an all-pay auction with complete information to model political campaigns, and show how a cap on individual political contribution may actually increase aggregate expenditures. A recent paper by $\mathrm{Fu}, \mathrm{Lu}$ and $\mathrm{Lu}$ (2009) analyzes how a principal should allocate a fixed budget between subsidies and prizes in order to motivate innovation. In Taylor (1995), Fullerton and McAfee (1999) and in Che and Gale (2003) research contests are modeled as rank-ordered tournaments and it is shown that it is beneficial to exclude some participants (see also Baye, Kovenock, and de Vries, 1993). In these papers exclusion is costless for the designer.

\footnotetext{
${ }^{3}$ Other papers that study the effects of multiple prizes in contests are Glazer and Hassin (1988), Barut and Kovenock (1998), Clark and Riis (1998) and Moldovanu and Sela (2006).

${ }^{4}$ This paper introduced the Barlow-Proschan (1966) order statistics results into the mechanism design literature.
} 
In a recent paper, Akerlof and Holden (2007) extend the analysis of Lazear and Rosen (1981) to the case with multiple prizes. They link the optimal prize structure to the form of utility functions, and show that the prize difference between two adjacent top players is often smaller than the prize difference between two adjacent bottom players. Their model is quite different from ours: they assume that the relationship between effort and performance is stochastic, and that agents are homogeneous in abilities. Moreover, these authors do not model punishments as negative prizes.

Finally, we want to note that the experimental literature on bonuses versus fines has mostly focused on principal-agent designs (e.g, Andreoni et al., 2003, and Fehr and Schmidt, 2007). It would be very interesting to experimentally study the effects of prizes and punishments also in contest situations where several agents compete with each other.

The rest of the paper is organized as follows. Section 2 describes the basic model framework and characterizes the equilibrium. Costly punishment is studied in Section 3. The analysis of costless punishment is contained in Section 4. There we divide the analysis into two parts, according to whether the contestants can exit the contest or not. Section 5 concludes.

\section{The Model}

We consider a contest with $n$ risk-neutral players where each player $j$ makes an effort $e_{j}$. For simplicity, we postulate a deterministic relation between effort and output, and assume these to be equal. Efforts are submitted simultaneously. An effort $e_{j}$ causes a cost of $e_{j} / a_{j}$, where $a_{j}$ is an ability parameter.

The ability (or type) of contestant $j$ is private information to $j$. Abilities are drawn independently of each other from the interval $[0,1]$ according to a distribution function $F$ that is common knowledge. We assume that $F$ has a continuous density $f=d F>0$.

The designer can allocate $n$ prizes to the $n$ players: $V_{1} \leq V_{2} \leq \cdots \leq V_{n}$. Each $V_{i}$ can be positive (a reward), zero or negative (a punishment). The contestant with the highest effort wins the first prize $V_{n}$, the contestant with the second highest effort wins the second prize $V_{n-1}$, and so on until all the prizes are allocated. The payoff of contestant $i$ who has ability $a_{i}$ and submits effort $e_{i}$ is $V_{j}-e_{i} / a_{i}$ if $i$ wins prize $j$.

Each contestant $i$ chooses her effort in order to maximize her expected payoff (given the other competitors' efforts and the values of the different prizes). The contest designer determines the number and size of prizes in order to maximize total expected effort $\sum_{i=1}^{n} e_{i}$. 
We use the following notations:

1. $A_{k, n}$ denotes $k$-th order statistic out of $n$ independent variables independently distributed according to $F$. Note that $A_{n, n}$ is the highest order statistic, and so on...

2. $F_{k, n}$ denotes the distribution of $A_{k, n}$, and $f_{k, n}$ denotes its density;

3. $E(k, n)$ denotes the expected value of $A_{k, n}$, where we set $E(0, n)=0$. Note that $E(n, n)$ is the expectation of the maximum, or highest order statistic, and so on...

\subsection{Equilibrium Derivation and Total Effort}

We focus here on a symmetric equilibrium. Let $\beta(a)$ denote the "bidding" strategy for the player with type $a$. This function relates ability to equilibrium effort. Assuming a symmetric equilibrium in strictly increasing strategies and applying the revelation principle, we can formulate the player's optimization problem as follows: player $j$ with ability $a$ chooses to behave as an agent with ability $s$ in order to solve the following problem:

$$
\max _{s} \sum_{i=1}^{n} F_{i}^{n}(s) V_{i}-\frac{\beta(s)}{a}
$$

where $F_{i}^{n}(s)$ denotes the probability that a player's type $s$ ranks exactly $i$-th lowest among $n$ random variables distributed according to $F$. The first term represent the expected payment from reporting $s$, while the second term is the effort cost. It is easy to verify that

$$
F_{i}^{n}(s)=\frac{(n-1) !}{(i-1) !(n-i) !}[F(s)]^{i-1}[1-F(s)]^{n-i}, i=1,2, \ldots, n .
$$

Define $F_{n, n-1}(s) \equiv 0$ and $F_{0, n-1}(s) \equiv 1$ for all $s \in[0,1]$. Then it is immediate that $F_{i}^{n}(s)=$ $F_{i-1, n-1}(s)-F_{i, n-1}(s)$. Therefore, we can rewrite the agent's maximization problem as

$$
\max _{s} \sum_{i=1}^{n}\left[F_{i-1, n-1}(s)-F_{i, n-1}(s)\right] V_{i}-\frac{\beta(s)}{a} .
$$

In equilibrium, the above maximization problem must be solved by $s=a$, and the solution of the resulting differential equation with boundary condition $\beta(0)=0$ is given by

$$
\beta(a)=\int_{0}^{a} x\left\{-f_{1, n-1}(x) V_{1}+\sum_{i=2}^{n-1}\left[f_{i-1, n-1}(x)-f_{i, n-1}(x)\right] V_{i}+f_{n-1, n-1}(x) V_{n}\right\} d x .
$$

The expected total effort is given by

$$
E_{\text {total }}=n \int_{0}^{1} \beta(a) f(a) d a .
$$


Note that

$$
\begin{aligned}
& n \int_{0}^{1}\left[\int_{0}^{a} x f_{r, n-1}(x) d x\right] f(a) d a \\
= & n\left[F(a) \int_{0}^{a} x f_{r, n-1}(x) d x\right]_{0}^{1}-n \int_{0}^{1} F(a) a f_{r, n-1}(a) d a \\
= & n \int_{0}^{1} a(1-F(a)) f_{r, n-1}(a) d a,
\end{aligned}
$$

where the first equality follows from integration by part. We further observe that

$$
n(1-F(a)) f_{r, n-1}(a)=(n-r) f_{r, n}(a) .
$$

Therefore, we have

$$
n \int_{0}^{1}\left[\int_{0}^{a} x f_{r, n-1}(x) d x\right] f(a) d a=(n-r) E(r, n) .
$$

By repeatedly applying above equation, the expected total effort becomes

$$
E_{\text {total }}=E_{\text {total }}\left(V_{1}, V_{2}, \ldots, V_{n}\right)=\sum_{i=1}^{n}[(n-i+1) E(i-1, n)-(n-i) E(i, n)] V_{i} .
$$

It is clear from equation (2) that the expected total effort $\left(E_{\text {total }}\right)$ is linear in prizes $V_{i}(i=1, . ., n)$. Thus, the optimal prize structure will depend on the magnitude of the different marginal effects of each prize.

\section{The Optimal Prize Structure with Costly Punishments}

We begin by studying the optimal prize structure when punishment is costly to the designer, and when the designer faces a fixed budget. Throughout this section, players have no option of quitting the contest. For example, in a soccer league, punishment may mean relegation to a lower league. Teams have no other option. Another realistic interpretation is that contestants prefer to participate in the contest even after taking into account the possibility of punishment. For example, in a labor market setting, punishment may mean a salary cut such that the worker is still willing to stay with the current employer instead of looking for another job or claiming unemployment benefits.

The designer has a budget $P<\infty$. The marginal cost of providing a unit of positive prize is normalized to 1, while the marginal cost of providing a unit of negative prize is denoted by $\gamma>0$. Without loss of generality, suppose there are $J(0 \leq J \leq n)$ negative prizes. That is, $V_{j}<0$ for 
$j \leq J$ and $V_{j} \geq 0$ for $j>J$. Then the designer's problem can be written as

$$
\begin{aligned}
& \max _{\left\{V_{1}, \ldots, V_{n}\right\}} E_{\text {total }}\left(V_{1}, V_{2}, \ldots, V_{n}\right) \\
\text { s.t. } \quad & \gamma \sum_{j=1}^{J}\left(-V_{j}\right)+\sum_{j=J+1}^{n} V_{j} \leq P .
\end{aligned}
$$

The designer chooses the prize structure to maximize total expected effort subject to the constraint that the monetary cost of rewards and punishments cannot exceed the budget $P$. Since both the objective function and the constraint are linear in prizes, the key objects are the marginal gains in expected total effort from increasing punishments or rewards.

We start with a new, simple proof of one of the main results in Moldovanu and Sela (2001): a winner-takes-all contest is optimal among all those that award only non-negative prizes (rewards) if the effort cost function is linear in effort input. Notice that it follows from (2) that, for $1 \leq i \leq n$,

$$
\frac{\partial E_{t o t a l}}{\partial V_{i}}=(n-i+1) E(i-1, n)-(n-i) E(i, n),
$$

which can be rewritten as

$$
\frac{\partial E_{t o t a l}}{\partial V_{i}}=E(i, n)-(n-i+1)[E(i, n)-E(i-1, n)]
$$

Proposition 1 If only rewards can be allocated, then, for every distribution of abilities $F$, the optimal prize structure is $V_{n}=P$ and $V_{i}=0$ for all $i<n$.

Proof. Note that from (3) we have

$$
\frac{\partial E_{t o t a l}}{\partial V_{n}}=E(n-1, n) \geq 0,
$$

and, for $1 \leq i<n$,

$$
\frac{\partial E_{t o t a l}}{\partial V_{n}}-\frac{\partial E_{t o t a l}}{\partial V_{i}}=E(n-1, n)-E(i, n)+(n-i+1)[E(i, n)-E(i-1, n)] \geq 0 .
$$

Thus, the designer optimally rewards only the player with the highest effort.

Next, we allow the designer to punish players with poor performance. It turns out that the optimal prize structure is then related to the curvature of the ability distribution $F$. A key concept for our analysis is the failure rate (or hazard rate) defined as follows:

Definition 1 The failure rate (or hazard rate) of a distribution $F$ is given by:

$$
\lambda(x)=\frac{f(x)}{1-F(x)}
$$

A distribution function $F$ has an increasing failure rate $(I F R)$ if its failure rate, $\lambda(x)$, is increasing. 
The following results, due to Barlow and Proschan (1966), link properties of order statistics to properties of the distribution $F$. We shall use these results repeatedly throughout the paper:

Lemma 1 (a). Let $F$ and $G$ be two distributions such that $F(0)=G(0)=0$, and let $G^{-1} F$ be convex on the support of $F$. Let $E_{F}(i, n)$ and $E_{G}(i, n)$ denote the expected value of the $i$-th order statistic out of $n$ independent variables independently distributed according to $F$ and $G$, respectively. Then $E_{F}(i, n) / E_{G}(i, n)$ is decreasing in $i$ for a fixed $n$.

(b). Assume that a distribution $F$ with $F(0)=0$ is convex (concave). Then $E(i, n) / i$ is decreasing (increasing) in $i$ for a fixed $n$.

(c). Assume that a distribution $F$ with $F(0)=0$ satisfies IFR. Then the random variable $(n-$ $i+1)\left(A_{i, n}-A_{i-1, n}\right)$ is stochastically decreasing in $i$ for a fixed $n$.

By applying Lemma 1 $(\mathrm{c})$, we can prove a counterpart to Proposition 1 for the case where only punishments are allowed:

Proposition 2 If only punishments can be allocated, and if the distribution of abilities $F$ satisfies IFR, then the optimal prize structure is $V_{1}=-\frac{P}{\gamma}$ and $V_{i}=0$ for all $i>1$.

Proof. If the distribution $F$ satisfies IFR, then, by Lemma 1. (c), $(n-i+1)\left(A_{i, n}-A_{i-1, n}\right)$ is stochastically decreasing in $i$, and thus $-(n-i+1)[E(i, n)-E(i-1, n)]$ is increasing in $i$. Since $E(i, n)$ always increases in $i$, it follows from 3 that $\frac{\partial E_{\text {total }}}{\partial V_{i}}$ is increasing in $i$ when $F$ satisfies IFR.

If the designer cannot use rewards, only negative terms of the form $\frac{\partial E_{\text {total }}}{\partial V_{i}}$ are relevant for the designer's optimal decision. Note that according to (3), for any distribution of abilities,

$$
\frac{\partial E_{t o t a l}}{\partial V_{1}}=-(n-1) E(1, n)<0 .
$$

Since $\frac{\partial E_{\text {total }}}{\partial V_{i}}$ is increasing in $i, \frac{\partial E_{\text {total }}}{\partial V_{1}}$ must have the highest absolute value among all negative terms. Consequently, it is most effective to only punish the player with the lowest effort.

It is important to note that, in contrast to Proposition 1 that holds for any distribution, Proposition 2 holds only under the $I F R$ requirement. In this case enough players are "threatened" by the highest punishment, which makes it quite effective. The following example illustrates that it may not be optimal to punish only the player with the lowest effort if the distribution of abilities does not satisfy IFR: 
Example 1 Let $F(x)=\sqrt[w]{x}$ and $\gamma=1$. Then $E(i, n)=\frac{n !(w+i-1) !}{(i-1) !(n+w) !}$. It is easy to verify that $F$ does not satisfy IFR. Note that it is not optimal to punish only the player with the lowest effort if

$$
\begin{aligned}
\frac{\partial E_{\text {total }}}{\partial V_{2}}-\frac{\partial E_{\text {total }}}{\partial V_{1}} & =2(n-1) E(1, n)-(n-2) E(2, n) \\
& =\frac{n ! w !}{(n+w) !}(2(n-1)-(w+1)(n-2))<0 .
\end{aligned}
$$

The above holds, for example, if $n=3$ and $w>3$.

We now proceed to investigate the optimal prize structure when both rewards and punishments are allowed. By inequality 4 , it is never optimal to allocate rewards to players other than the top performer. If the distribution $F$ satisfies $I F R$, then $\frac{\partial E_{\text {total }}}{\partial V_{1}}$ has the highest absolute value among all negative terms (see proof of Proposition 2), and therefore the optimal prize structure hinges on the comparison between the effectiveness of the top reward and the bottom punishment. Thus , we need to compare the absolute value of $\frac{\partial E_{\text {total }}}{\partial V_{1}}=-(n-1) E(1, n)$ with $\gamma \frac{\partial E_{\text {total }}}{\partial V_{n}}=\gamma(E n-1, n)$

Proposition 3 Suppose that the distribution of abilities F satisfies IFR.

1. If $(n-1) E(1, n)>\gamma E(n-1, n)$, then the optimal prize structure is $V_{1}=-\frac{P}{\gamma}$ and $V_{i}=0$ for all $i>1$.

2. If $(n-1) E(1, n) \leq \gamma E(n-1, n)$, then the optimal prize structure is $V_{n}=P$ and $V_{i}=0$ for all $i<n$.

As a special case, let us assume for the next result that the marginal cost of rewards and punishments is the same, that is $\gamma=1$. We obtain then:

Proposition 4 Assume that $\gamma=1$.

1. If the distribution of abilities $F$ is convex, the optimal prize structure is $V_{1}=-P$ and $V_{i}=0$ for all $i>1$.

2. If the distribution of abilities $F$ is concave and satisfies IFR, then the optimal prize structure is $V_{n}=P$ and $V_{i}=0$ for all $i<n$.

3. If $F$ is concave $!^{5}$ then the optimal prize structure may include up to $\frac{n}{2}$ punishments.

\footnotetext{
${ }^{5}$ Note that a concave function may or may not satisfy IFR.
} 
Proof. 1) By Lemma 1 (b), if $\mathrm{F}$ is convex then $E(i, n) / i$ is decreasing in $i$. Thus, we have

$$
(n-1) E(1, n)>E(n-1, n) .
$$

The result follows from Proposition 3 , since $F$ convex implies that $F$ satisfies IFR.

2) If $F$ is concave then $E(i, n) / i$ is increasing in $i$ (Lemma 1 -(b)). In particular, $(n-1) E(1, n)<$ $E(n-1, n)$, and the result follows from Proposition 3 .

3) Lemma 1 - (b) yields that

$$
\frac{E(i-1, n)}{E(i, n)}<\frac{i-1}{i}, \forall i
$$

Note that

$$
\frac{i-1}{i}<\frac{n-i}{n-i+1} \text { if and only if } i<\frac{n+1}{2}
$$

This yields

$$
(n-i+1) E(i-1, n)<(n-i) E(i, n) \text { for } i<\frac{n+1}{2} .
$$

Therefore,

$$
\frac{\partial E_{t o t a l}}{\partial V_{i}}=(n-i+1) E(i-1, n)-(n-i) E(i, n)<0, \text { for } i<\frac{n+1}{2} .
$$

Note that the uniform distribution is both convex and concave, and that it satisfies IFR. Assuming that the marginal costs of rewards and punishments are the same, the above proposition implies that, with a uniform distribution of abilities, it is optimal to either punish the worst performer only or to reward the best performer only. The effectiveness of punishing the player with the lowest effort equals then the effectiveness of rewarding the player with the highest effort.

In certain applications, all punishments have a fixed value for the agents and a fixed cost for the designer. For example, think of a firm who can choose the number of workers to fire in a recession: it is reasonable to assume that the cost of each layoff is fixed for the firm (e.g., some compensation that needs to be paid to each fired worker), and for the workers themselves (e.g, the difference between a fixed wage and a lower unemployment aid). In this case we show that punishments and rewards co-exist in the optimal solution.

We assume that there are $q$ punishments, each yielding utility $-U \leq 0$ to agents and causing a cost $\gamma U$ to the designer. Since we have shown that positive rewards, if any, should be assigned to the top performer only, we will assume here that only the player with the highest effort is rewarded by a prize $V_{n} \geq 0$. We also assume that the distribution of $F$ satisfies $I F R$. 
Since the designer is not able to shift the entire budget to one, big punishment for the agent with the lowest performance, and since the value/cost of punishments is fixed, the interesting question here is how many punishments are optimal (if any). The designer's optimization problem becomes then

$$
\begin{aligned}
& \max _{\left\{q, V_{n}\right\}} \sum_{i=1}^{q}[(n-i+1) E(i-1, n)-(n-i) E(i, n)](-U)+E(n-1, n) V_{n} \\
& \text { s.t. } q \gamma U+V_{n} \leq P .
\end{aligned}
$$

Notice that, by $3, \frac{\partial E_{\text {total }}}{\partial V_{i}}$ is increasing in $i$ when $F$ satisfies IFR. If

$$
\left|\frac{\partial E_{\text {total }}}{\partial V_{1}}\right| \leq \gamma\left|\frac{\partial E_{\text {total }}}{\partial V_{n}}\right|
$$

then it is optimal to have no punishments: $q=0$, and $V_{n}=P$.

Otherwise, let $i^{*}$ be the highest index such that

$$
\frac{\partial E_{\text {total }}}{\partial V_{i^{*}}} \leq 0 \text { and }\left|\frac{\partial E_{\text {total }}}{\partial V_{i^{*}}}\right|>\gamma\left|\frac{\partial E_{\text {total }}}{\partial V_{n}}\right| .
$$

Since $\frac{\partial E_{\text {total }}}{\partial V_{i}}$ is increasing in $i$, and since $\left|\frac{\partial E_{\text {total }}}{\partial V_{1}}\right|>\gamma\left|\frac{\partial E_{\text {total }}}{\partial V_{n}}\right|, i^{*}$ is well defined. Note that for all $1 \leq i \leq i^{*}$ it must hold that $\frac{\partial E_{\text {total }}}{\partial V_{i}} \leq 0$. Thus, it is optimal to set $i^{*}$ punishments: $q=i^{*}$ and $V_{n}=P-i^{*} \gamma U$.

Therefore, we have proved the following result:

Proposition 5 Assume that $F$ satisfies IFR, and suppose the designer has to incur a positive cost $\gamma U$ for each punishment which yields a fixed negative payoff $-U$ to agents.

1. If condition (5) holds, then no punishment $\left(q=0\right.$ and $\left.V_{n}=P\right)$ is optimal.

2. If condition (5) does not hold, then it is optimal to set $q=i^{*}$ punishments, with $V_{n}=P-i^{*} \gamma U$ and $i^{*}$ defined in (6).

\section{The Optimal Prize Structure with Costless Punishments}

We assume now that punishments are costless, that is, $\gamma=0$. It is obvious that as long as the designer need not worry about the participation constraint, punishments will necessarily be part of the optimal incentive scheme. Moreover, since punishments are not costly, the available budget is best allocated to rewarding the top performer (only). 


\subsection{Contests without exit}

We assume here that no player has the option to stay out of the contest. Punishing the bottom player always increases the total effort. Assuming a fixed punishment $-U$, we look then for the optimal number of identical, costless punishment: Denote by $q \geq 1$ the number of punishments. By (2), total effort is given by

$$
E_{\text {total }}=V_{n} E(n-1, n)-U(n-q) E(q, n) .
$$

The following proposition summarizes a general relation between the optimal number of punishments and the distribution of the players' abilities.

Proposition 6 Assume that $\gamma=0$, and consider two distributions of abilities $G, F$ leading to optimal numbers of punishments $q_{G}$ and $q_{F}$, respectively. If $G^{-1} F$ is convex then $q_{G} \geq q_{F}$.

Proof. Since $q_{F}$ is optimal for $F$, we have for all $q<q_{F}$,

$$
\left(n-q_{F}\right) E_{F}\left(q_{F}, n\right) \geq(n-q) E_{F}(q, n) \Leftrightarrow \frac{E_{F}\left(q_{F}, n\right)}{E_{F}(q, n)} \geq \frac{(n-q)}{\left(n-q_{F}\right)} .
$$

In order to show that $q_{G} \geq q_{F}$, it is sufficient to show that, under the distribution $G$, total effort induced by $q_{F}$ punishments is larger than total effort induced by $q<q_{F}$ punishments. That is, we need to show that, for $q<q_{F}$,

$$
\left(n-q_{F}\right) E_{G}\left(q_{F}, n\right) \geq(n-q) E_{G}(q, n) \Leftrightarrow \frac{E_{G}\left(q_{F}, n\right)}{E_{G}(q, n)} \geq \frac{(n-q)}{\left(n-q_{F}\right)}
$$

Thus, it is sufficient to show that for $q<q_{F}$,

$$
\frac{E_{F}(q, n)}{E_{G}(q, n)} \geq \frac{E_{F}\left(q_{F}, n\right)}{E_{G}\left(q_{F}, n\right)}
$$

The last inequality follows from Lemma 1. (a).

A simple corollary is:

Corollary 1 Assume that $\gamma=0$. If the distribution of abilities $F$ is convex (concave), then the optimal number of punishments is smaller (larger) than $n / 2$.

Proof. Let $F$ be the uniform distribution. Total effort is given by

$$
E_{\text {total }}=V_{n} \frac{n-1}{n+1}-U(n-q) \frac{q}{n+1}
$$

\footnotetext{
${ }^{6}$ If punishment is not costly, agents cannot exit, and punishment is arbitrary, the problem is of course trivial.
} 
The optimal number of punishments $q$ must satisfy

$$
\frac{d E_{t o t a l}}{d q}=\frac{-U}{n+1}(n-2 q)=0 .
$$

Thus $q=n / 2$. The result follows then by Proposition 6 .

When only a few players are punished, high ability contestants know that they can avoid punishment with sufficiently high probability by exerting effort. Conversely, if the designer punishes most players, high ability players get discouraged: they may get punished even if they work hard. The above result follows intuitively from this observations since a convex (concave) distribution puts more (less) weight on high abilities than a uniform one.

\subsection{Contests with exit}

We consider now the case where only types with positive expected payoffs participate in the contest, while the other players stay out. We assume that there is one reward $V_{n} \geq 0$, and $q \geq 1$ punishments, each equal to $V_{1} \geq-V_{n}$. If only one player participates, we assume that he receives a reduced prize worth $V_{n}+V_{1}$.

Let $a^{*}$ denote the type of a player who is indifferent between attending the contest and staying out. The maximization problem of a player with type $a$ is

$$
\max _{s} V_{n} F_{n}^{n}(s)+V_{1} \sum_{i=1}^{q} G_{i}^{n}(s)-\frac{\beta(s)}{a}
$$

where

$$
G_{i}^{n}(s)=\left(\begin{array}{c}
n-1 \\
i-1
\end{array}\right)\left(F(s)-F\left(a^{*}\right)\right)^{i-1}\left[1-F(s)+F\left(a^{*}\right)\right]^{n-i},
$$

is the probability that a player with type $s$ ranks $i$-th lowest among the players who enter the contest. Let

$$
G(s)=F(s)-F\left(a^{*}\right) .
$$

Then, we can rewrite $G_{i}^{n}(s)$ as

$$
G_{i}^{n}(s)=\left(\begin{array}{c}
n-1 \\
i-1
\end{array}\right) G(s)^{i-1}(1-G(s))^{n-i} .
$$

If a player with type $a^{*}$ enters the contest then, by the definition of $a^{*}$, she gets for sure the negative prize $V_{1}$ and wins the positive prize $V_{n}$ only if all other players have lower types. That is, the cutoff $a^{*}$ is given by

$$
V_{n} F\left(a^{*}\right)^{n-1}=-V_{1}
$$


The necessary first-order condition for an agent's maximization problem is

$$
V_{n} f_{n-1, n-1}(a)-V_{1}(n-1)\left(\begin{array}{c}
n-2 \\
q-1
\end{array}\right) G(a)^{q-1}(1-G(a))^{n-q-1} f(a)-\frac{\beta^{\prime}(a)}{a}=0
$$

To simplify notation, define

$$
\phi_{q, n-1}(s)=(n-1)\left(\begin{array}{c}
n-2 \\
q-1
\end{array}\right) G(s)^{q-1}[1-G(s)]^{n-q-1} .
$$

Then, the equilibrium bidding function for a bidder with type $a \geq a^{*}$ is

$$
\beta(a)=\int_{a^{*}}^{a}\left[V_{n} s f_{n-1, n-1}(s)-V_{1} s \phi_{q, n-1}(s) f(s)\right] d s
$$

where we use the fact that $\beta\left(a^{*}\right)=0$. Thus, total effort is given by

$$
E_{\text {total }}=n \int_{a^{*}}^{1} \beta(a) f(a) d a .
$$

Interestingly, even though the players are allowed to quit the contest, punishments have a role to play in maximizing the expected total effort from the participating players, analogously to the beneficial role of an entry fee or minimal effort requirement.

Proposition 7 Assume that $\gamma=0$, and assume that agents have the option not to participate in the contest, which yields zero utility. Then, it is always optimal to punish, i.e., $V_{1}<0$.

Proof. Since $a^{*}$ is given by $V_{n} F\left(a^{*}\right)=-V_{1}$ we obtain

$$
V_{n} f\left(a^{*}\right) \frac{\partial a^{*}}{\partial V_{1}}=-1 \Leftrightarrow f\left(a^{*}\right) \frac{\partial a^{*}}{\partial V_{1}}=-\frac{1}{V_{n}}
$$

Let us normalize $V_{n}=1$. Total effort becomes then

$$
E_{\text {total }}=n \int_{a^{*}}^{1}\left\{\int_{a^{*}}^{a}\left[s f_{n-1, n-1}(s)-V_{1} s \phi_{q, n-1}(s) f(s)\right] d s\right\} f(a) d a
$$

Taking the derivative with respect to $V_{1}$ yields

$$
\begin{aligned}
\frac{\partial E_{\text {total }}}{\partial V_{1}}= & -n\left\{\int_{a^{*}}^{a^{*}}\left[s f_{n-1, n-1}(s)-V_{1} s \phi_{q, n-1}(s) f(s)\right] d s\right\} f\left(a^{*}\right) \frac{\partial a^{*}}{\partial V_{1}} \\
& +n \int_{a^{*}}^{1} \frac{\partial\left\{\int_{a^{*}}^{a}\left[s f_{n-1, n-1}(s)-V_{1} s \phi_{q, n-1}(s) f(s)\right] d s\right\}}{\partial V_{1}} f(a) d a
\end{aligned}
$$

Note that

$$
\begin{aligned}
& \frac{\partial\left\{\int_{a^{*}}^{a}\left[s f_{n-1, n-1}(s)-V_{1} s \phi_{q, n-1}(s) f(s)\right] d s\right\}}{\partial V_{1}} \\
= & -\left[a^{*}(n-1) F\left(a^{*}\right)^{n-2} f\left(a^{*}\right)-V_{1} a^{*} \phi_{q, n-1}\left(a^{*}\right) f\left(a^{*}\right)\right] \frac{\partial a^{*}}{\partial V_{1}}-\int_{a^{*}}^{a} s \phi_{q, n-1}(s) f(s) d s \\
= & a^{*}(n-1) F\left(a^{*}\right)^{n-2}-V_{1} a^{*} \phi_{q, n-1}\left(a^{*}\right)-\int_{a^{*}}^{a} s \phi_{q, n-1}(s) f(s) d s
\end{aligned}
$$


The last equality uses $f\left(a^{*}\right) \frac{\partial a^{*}}{\partial V_{1}}=-1$.

Therefore,

$$
\frac{\partial E_{\text {total }}}{\partial V_{1}}=n \int_{a^{*}}^{1}\left\{a^{*}(n-1) F\left(a^{*}\right)^{n-2}-V_{1} a^{*} \phi_{q, n-1}\left(a^{*}\right)-\int_{a^{*}}^{a} s \phi_{q, n-1}(s) f(s) d s\right\} f(a) d a
$$

By condition (8), when $V_{1}=0$ we must have $a^{*}=0$. Thus

$$
\left.\frac{\partial E_{\text {total }}}{\partial V_{1}}\right|_{V_{1}=0}=-n \int_{0}^{1}\left\{\int_{0}^{a} s \phi_{q, n-1}(s) f(s) d s\right\} f(a) d a<0 .
$$

Therefore, punishments are always efficient even in the contest with exit.

Example 2 Consider a contest with two players who have the option not to participate. Obviously, at most one player can be then punished. Noting that $\phi_{1,1}(s)=1$, we get $\beta(a)=$ $\int_{a^{*}}^{a}\left(V_{n}-V_{1}\right) s f(s) d s$, where $a^{*}$ is given by $V_{n} F\left(a^{*}\right)=-V_{1}$. Total effort is given by

$$
\begin{aligned}
E_{\text {total }} & =2 \int_{a^{*}}^{1}\left[\int_{a^{*}}^{a}\left(V_{n}-V_{1}\right) s f(s) d s\right] f(a) d a \\
& =2 \int_{F^{-1}\left(\frac{-V_{1}}{V_{n}}\right)}^{1}\left[\int_{F^{-1}\left(\frac{-V_{1}}{V_{n}}\right)}^{c}\left(V_{n}-V_{1}\right) s f(s) d s\right] f(a) d a .
\end{aligned}
$$

Suppose now that $F$ is uniform on $[0,1]$, and let $V_{n}=1$. Then

$$
E_{\text {total }}=2 \int_{-V_{1}}^{1}\left(\int_{-V_{1}}^{a}\left(1-V_{1}\right) s d s\right) d a=\frac{1}{3}\left(V_{1}+1\right)^{2}\left(2 V_{1}^{2}-3 V_{1}+1\right),
$$

which is maximized for $V_{1}=-0.16$.

Finally, we want to note that, in some situations, other mechanisms for exclusion may perform better than punishments. For example, punishments that are not pecuniary (i.e., negative prizes that are not paid to the designer) are dominated by a contest with an entry fee that the designer can collect. This can be easily seen by setting an entry fee that excludes the same set of types as a given negative priz€

\section{Concluding Remarks}

We have investigated the combined effect of prizes and punishments in contests, and we derived the optimal prize structures in several environments with both costly and costless punishment. Our main results link the prize structure to features of the distribution of ability in the population, and

\footnotetext{
${ }^{7}$ Both instruments are used in practice: European sports leagues use relegation for punishing teams that do not perform, while US professional leagues demand entry (franchise) fees.
} 
to the marginal costs of rewards versus punishments. In particular, we give conditions under which rewarding the top performers is more (less) effective than punishing the worst performers. Finally, we have shown that punishments may have a beneficial effect on effort even if contestants have the option not to participate. Our present analysis completes the picture against the background of a large literature that has only considered positive prizes (rewards).

\section{References}

1. Akerlof, R. and H. Richard (2007), "The Nature of Tournaments," working paper, Sloan School of Management, MIT.

2. Andreoni J., W. Harbaugh and L. Vesterlund (2003), "The Carrot or the Stick: Rewards, Punishments, and Cooperation," American Economic Review 93, 893-902.

3. Arvey, R. D. and J. M. Ivancevich (1980), "Punishment in Organizations: A Review, Propositions, and Research Suggestions. Academy of Management Review 5, 123-132.

4. Barlow, R. and F. Proschan (1966), "Inequalities for Linear Combinations of Order Statistics from Restricted Families," Ann. Math. Statistic. 37, 1593-1601.

5. Baye, M., Kovenock, D., and de Vries, C. (1993), "Rigging the Lobbying Process," American Economic Review, 83, 289-294.

6. Barut, Y., and Kovenock, D. (1998), "The Symmetric Multiple Prize All-Pay Auction with Complete Information," European Journal of Political Economy, 14, 627-644.

7. Chan, W., P. Courty and H. Li. (2010), "Suspense," Economic Journal, forthcoming.

8. Che, Y., and Gale, I. (2003), "Optimal Design of Research Contests," American Economic Review, 93, 646-671.

9. Clark, D., and Riis, C. (1998), "Competition over More than One Prize," American Economic Review, 88, 276-289.

10. Fehr, E., and Schmidt, K.M. (2007), "Adding a Stick to the Carrot? The Interaction of Bonuses and Fines", American Economic Review, 97, 177-181.

11. Fu, Q., J. Lu and Y. Lu (2009), "How to Motivate Innovation: Subsidies and Prizes?" working paper, National University of Singapore. 
12. Fullerton, R., and R. P. McAfee, "Auctioning Entry into Tournaments," Journal of Political Economy, June 1999, 107(3), 573-605.

13. Glazer, A., and Hassin, R. (1988), "Optimal Contests," Economic Inquiry, 26, 133-143.

14. Green, J., and N. Stokey (1983), "A Comparison of Tournaments and Contracts," Journal of Political Economy 91(3): 349-364.

15. Lazear, E. and S. Rosen (1981), "Rank-Order Tournaments as Optimum Labor Contracts," Journal of Political Economy 89(5): 841-864.

16. Moldovanu, B. and A. Sela (2001), "The Optimal Allocation of Prizes in Contests," American Economic Review 91(3), 542-558.

17. Moldovanu, B. and A. Sela (2006), "Contest Architecture," Journal of Economic Theory 126(1): 70-97.

18. Moldovanu, B., A. Sela and X. Shi (2007), "Contests for Status," Journal of Political Economy, $115(2), 338-363$.

19. Nalebuff, B. and J. Stiglitz (1983), "Prizes and Incentives: Towards a General Theory of Compensation and Competition," Bell Journal of Economics 14(1): 21-43.

20. Taylor C. R., "Digging for Golden Carrots: An Analysis of Research Tournaments," American Economic Review, 1995, 85 (4), 872-890. 